\title{
Avian Beta Defensin 2 (AvBD2) Gene Polymorphism Identification in IPB-D1 Chicken
}

\author{
Masrurah $^{1}$, Khaerunnisa $I^{2}$, Murtini $S^{3}$, Sumantri $C^{1}$ \\ ${ }^{1}$ Department of Animal Production and Technology, Faculty of Animal Science, IPB University, Bogor 16680 \\ ${ }^{2}$ Research Center for Biotechnology, Indonesian Institute of Sciences, Bogor 16911 \\ ${ }^{3}$ Department of Animal Disease and Veterinary Public Health, Faculty of Veterinary Medicine, IPB University, Bogor 16680 \\ E-mail: csumantri12@gmail.com; ceces@ipb.ac.id
}

(received 10-03-2021; revised 28-05-2021; accepted 21-06-2021)

\begin{abstract}
ABSTRAK
Masrurah, Khaerunnisa I, Murtini S, Sumantri C. 2020. Identifikasi keragaman gen Avian Beta Defensin 2 (AvBD2) pada ayam IPB-D1. JITV 26(2): 82-89. DOI: http://dx.doi.org/10.14334.v26i2.2715.

Gen Avian Beta Defensin 2 (AvBD2) yang berada di kromosom 3 berperan penting dalam sistem imunitas tubuh ayam dengan menghambat perkembangan mikroorganisme seperti bakteri yang mengifeksi jaringan tubuh. Defensin dihasilkan melalui sel epitel segera setelah adanya cidera jaringan atau infeksi, yang kemudian memproses pematangan sel dendritik untuk memulai respon imun di kelenjar getah bening. Tujuan penelitian ini adalah mengidentifikasi polimorfisme gen AvBD2 pada ayam IPB-D1. Metode PCR dan direct-DNA sequencing digunakan untuk mengidentifikasi keragaman intron 1, ekson 2, dan intron 2 gen AVDB2 pada 47 ekor ayam IPB-D1. Perhitungan frekuensi genotipe, frekuensi alel, dan heterozigositas dilakukan untuk mendapatkan informasi polimorfisme gen AvBD2. Sebanyak 10 mutasi titik ditemukan pada gen AvBD2 yang tersebar di intron 1 (g.4843T>A, g.4853G $>$ A, dan g.4859T $>$ C), ekson 2 (g.4881A $>$ G, g.4889G $>$ A, dan g.5002C $>$ T), dan intron 2 (g.5075C $>$ T, g.5111T $>$ G, g.5116G $>$ T, dan g.5177G $>$ T). Seluruh SNP bersifat polimorfik. Mutasi g.5002C $>$ T menyebabkan perubahan asam amino Ala menjadi Val yang berpotensi menjadi kandidat penciri ketahanan penyakit pada ayam IPB-D1.
\end{abstract}

Kata Kunci: Gen AvBD2, IPB-D1 chicken, Keragaman

\section{ABSTRACT}

Masrurah, Khaerunnisa I, Murtini S, Sumantri C. 2020. Avian Beta Defensin 2 (AvBD2) gene polymorphism identification in IPB-D1 chicken. JITV 26(2): 82-89. DOI: http://dx.doi.org/10.14334.v26i2.2715.

Avian Beta Defensin 2 (AvBD2) gene, located in chromosome 3, plays an important role in the immune system of the chicken by inhibiting the development of microorganisms such as bacteria that infect body tissues. Defensins are produced through epithelial cells immediately after tissue injury or infection, which then processes the maturation of dendritic cells to initiate an immune response in the lymph nodes. The purpose of this study was to discover the polymorphism of the AvBD2 gene in IPB-D1 chickens. PCR and direct-DNA sequencing methods were used to identify the polymorphisms of intron 1, exon 2 , and intron $2 \mathrm{AvDB} 2$ genes in 47 chickens. Genotype and allele frequency, and heterozygosity calculations were carried out to obtain information of the AvBD2 gene polymorphism. A total of 10 single nucleotide polymorphisms were found in the AvBD2 gene located in intron 1 (g.4843T $>$ A, g.4853G $>A$, and g.4859T $>C$ ), exon 2 (g.4881A $>$ G, g.4889G $>$ A, and g.5002C $>$ T), and intron 2 (g.5075C $>\mathrm{T}$, g.5111T $>\mathrm{G}$, g. $5116 \mathrm{G}>\mathrm{T}$, and g.5177G $>\mathrm{T}$ ). All SNPs are polymorphic. The g.5002C $>\mathrm{T}$ mutation causes changes in the amino acid Ala to Val which has the potential to be a candidate for characterizing disease resistance in IPB-D1 chickens.

Key Words: AvBD2 gene, IPB-D1 chicken, Polymorphism

\section{INTRODUCTION}

Indonesia's native chicken population in 2020 is estimated to reach 308 million chickens, or around $8.8 \%$ of the total national chicken population (DGAHP 2020). Application of extensive traditional rearing system shows the low application of biosecurity and sanitation. The lack of attention of local chicken breeders

*all authors contributed equally to the work as main contributors breeders to good rearing management and the application of biosecurity affect the health of local chickens. This condition causes local chicken production to be not optimal. Availability of local chicken breeds that are resistant to disease is very important so that breeders could get disease-resistant chickens maintained in suboptimal biosecurity and sanitation conditions. One of the efforts to provide these breeds is through genetic selection of local chickens that have a good immune system. 
Defensin is part of the immune system that has broad antimicrobial activity (Wilson et al. 2013). Beta defensin is a member of the defensins family found in avian including chickens and has the potential as an alternative to antibiotics due to its role to neutralize various pathogens such as colibacillosis and zoonosis (Derache et al. 2012, Kim \& Lillehoj 2019, Zhao et al. 2014). Hong et al. (2012) stated that the pathogens that phagocytes by antimicrobial activity in the AvBD2 gene include Salmonella enterica serovar Typhimurium, C. perfringens, and Escherichia coli found in the digestive tract. Moreover, the AvBD2 gene plays an important role in the level of immunity in chickens (Hong et al. 2012). AvDB2 has been reported to play a role in ileum and cecum mucosal defense in broiler embryos and neonatal chicks (Terada et al 2018). The AvBD2 gene was isolated from leukocytes, epithelial cells of the skin, digestive tract, and respiratory tract. The role of $\mathrm{AvBD} 2$ in resistance to Newcastle Disease virus infection was reported by Liu et al. (2018). Several molecules have also been reported to suppress the action of AvBD2 in defense against NDV, one of which is p38 MAPK (Liu et al. 2018). In addition, localization of AvBD2 and AvBD4 was found in chickens that were vaccinated against ND and Marek's disease (Shimizu et al. 2020).

Defensins are produced immediately after tissue injury or infection. Defensins are released from epithelial cells, including keratinocytes and infiltrating leukocytes. Defensins facilitate the uptake of antigens and processing of immature dendritic cells (iDCs) into mature dendritic cells (mDCs). These mDCs then migrate to the lymph nodes to initiate an immune response (Hazlett \& Wu 2011). The chicken AvBD2 gene is located on chromosome 3 and consists of four exons and three introns (Xiao et al. 2004). AvBD2 protein in chickens consists of 22 signal peptides, 6 propieces, and 36 mature peptides, which are equal to other avian such as ostrich, duck, goose, and turkey $(\mathrm{Lu}$ et al. 2014).

The polymorphisms of the AvBD2 gene is indicated by the discovery of single nucleotide polymorphysms (SNP) which can be used as genetic markers. Bagnicka et al. (2010) stated that SNPs on Avian beta defensin have a strong correlation to be associated with antibacterial activity against Gram-positive and Gramnegative bacteria. Mommarazi and Habibi (2017) reported that the mutation rate of chicken AvBD2 was 15.4 SNPs/kb, which showed that in every $1000 \mathrm{bp}$ of AvBD2 gene sequence, 15.4 SNPs were found. This shows a high genetic diversity and has a high response to disease resistance (Morammazi \& Habibi 2017).

IPB-D1 chicken is Indonesian local chickens that have been released by the Minister of Agriculture of the Republic of Indonesia in 2019 as superior broiler and layer (Sumantri et al. 2020). IPB-D1 chickens are the result of crossing of Pelung $\mathrm{x}$ Sentul (male) and Kampung x Cobb strain broilers (female), so they have a blood composition of $75 \%$ local chickens (Sumantri et al. 2020). Apart from having superior productivity, IPBD1 chickens are also developed to have resistance to ND virus (Al-Habib et al. 2020) and Salmonella enteritidis infection (Susanti et al. 2020). Genetic information regarding the diversity of the AVBD2 gene in IPB-D1 chickens has never been reported. The objective of this study is to discover the Single Nucleotide Polymorphism (SNP) of the partial AvBD2 gene in IPB-D1 chicken using direct-DNA sequencing method. Identification of the polymorphism of the AvBD2 gene may be a potential candidate gene for improving genetic quality in IPB-D1 chickens, especially in their immune system.

\section{MATERIALS AND METHODS}

\section{Chickens rearing management and blood sample collection}

All procedures performed in this study were based on animal research ethics (IPB Animal Care and Use Committee IACUC approval ID: 163-2019). A total of 47 IPB-D1 chickens were used for this study. All chickens were provided by the Animal Genetics and Breeding Division, Faculty of Animal Science, IPB University, Indonesia. Feeding was given twice a day. Chickens were fed $100 \%$ commercial feed, $70 \%$ commercial feed and $30 \%$ rice bran, and $60 \%$ commercial feed and $40 \%$ rice bran for starter $(0-4$ weeks), grower (4-12 weeks), and finisher (12-21 weeks), respectively. Clean drinking water is always available. The ND vaccine was administrated to chickens at 3 and 7 days old. At 21 weeks old, blood samples were collected from axillary vein on the wing.

\section{Primer designing}

Sequence data for primer designing was obtained from the National Center for Biotechnology Information (NCBI) with the Gen Bank access number AY621317.1 (Xiao et al. 2004). The primer sequences in this study is: forward (F): 5'CCCACAGAGCATCCATGAGG-3' and reverse (R): 5'-TTGCTGTTGTTGCAGGGTTG-3'. These primers produced a 411 bp DNA sequence covering partial intron 1 to intron 2 . The primers were designed using the Primer Designing Tool application (http://www.ncbi.nlm.nih.gov/tools/primer-blast/).

\section{DNA isolation and amplification}

To obtain genomic DNA template, the blood DNA extraction procedure was performed using a Blood/Cell 
DNA Mini Kit (Geneaid) according to the manufacturer's instructions. The amplification of AVBD2 gene fragments was carried out using a PCR machine (Gene Amp PCR System 9700, Applied Bio Systems) in a total of $23 \mu \mathrm{L}$ premix consisting of $2 \mu \mathrm{L}$ DNA template, $0.3 \mu \mathrm{L}$ primers, $12.5 \mu \mathrm{L}$ GoTaq ${ }^{\circledR}$ Green Master Mix (Promega), and 8.2 $\mu \mathrm{L}$ Nuclease-free Water. The PCR conditions used consisted of three stages. The first stage was the pre-denaturation process ( $95^{\circ} \mathrm{C}$ for 5 minutes). The second stage carried out 35 cycles consisting of denaturation $\left(95^{\circ} \mathrm{C}\right.$ for $\left.10 \mathrm{sec}\right)$, annealing $\left(62^{\circ} \mathrm{C}\right.$ for $\left.20 \mathrm{sec}\right)$, and extension $\left(72{ }^{\circ} \mathrm{C}\right.$ for $30 \mathrm{sec})$. The third stage is the final extension $\left(72^{\circ} \mathrm{C}\right.$ for 5 minutes). PCR products were visualized in $1.5 \%$ agarose gel using PeqGreen DNA/RNA Dye (VWR) staining under UV light (Alpha Imager, Alpha Innotech). Direct sequencing was then carried out for each sample using the $1^{\text {st }}$ Base sequencing services in Selangor, Malaysia.

\section{Data analysis}

The sequencing results in the form of a chromatogram were then analyzed using the MegaX
(Kumar et al. 2018) and BioEdit (Hall et al. 2011) for DNA sequence alignment and for DNA sequence visualization, respectively. Genetic diversity was analyzed by calculating genotype frequency, allele frequency, and heterozygosity values based on Nei \& Kumar (2000)

\section{RESULTS AND DISCUSSION}

\section{Identification of mutations in partial AVBD2 gene}

This study has successfully amplified the $411 \mathrm{bp}$ partial AvBD2 gene in IPB-D1 chickens (Figure 1). A total of 10 SNPs were found in intron 1 (3 SNPs), exon 2 (3 SNPs), and intron 2 (4 SNPs) of the AvBD2 gene (Table 1). One of the mutations in exon 2 was also reported by Morammazi \& Habibi (2017) in local Iranian chicken populations, i.e. g.5002C $>\mathrm{T}$. Meanwhile, the other nine mutations have never been reported previously. All mutations found, the types of mutations and amino acid changes can be found in Table 1

Six out of ten SNPs found were classified as transition mutations, i.e g.4853G $>$ A, g.4859T $>C$,

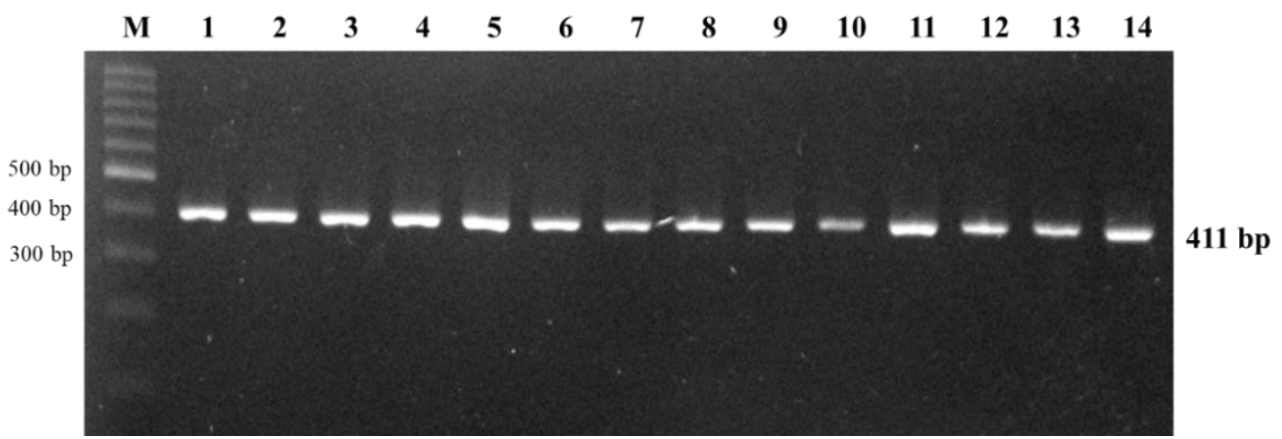

Figure 1. PCR result of partial AvBD2 gene in IPB-D1 chicken. $M=$ DNA ladder 100 bp; 1,2,..14 = sampel

Table 1. Point mutations found in intron 1 to intron 2 of the AvBD2 gene in IPB-D1 chickens along with the amino acid changes occurred

\begin{tabular}{|c|c|c|c|c|}
\hline No. & Mutation Position & Location in AvBD2 & Mutation Type & Amino Acid (Codon) Change \\
\hline 1. & g. $4843 \mathrm{~T}>\mathrm{A}$ & Intron 1 & Transversion & - \\
\hline 2. & g. $4853 \mathrm{G}>\mathrm{A}$ & Intron 1 & Transition & - \\
\hline 3. & g. $4859 \mathrm{~T}>\mathrm{C}$ & Intron 1 & Transition & - \\
\hline 4. & g. $4881 \mathrm{~A}>\mathrm{G}$ & Exon 2 & Transition & - \\
\hline 5. & g. $4889 \mathrm{G}>\mathrm{A}$ & Exon 2 & Transition & - \\
\hline 6. & g. $5002 C>T^{*}$ & Exon 2 & Transition & Ala (GCT) to Val (GTT) \\
\hline 7. & g. $5075 \mathrm{C}>\mathrm{T}$ & Intron 2 & Transition & - \\
\hline 8. & g. $5111 \mathrm{~T}>\mathrm{G}$ & Intron 2 & Transversion & - \\
\hline 9. & g. $5116 \mathrm{G}>\mathrm{T}$ & Intron 2 & Transversion & - \\
\hline 10. & g. $5177 \mathrm{G}>\mathrm{T}$ & Intron 2 & Transversion & - \\
\hline
\end{tabular}

*also reported by Morammazi and Habibi (2017) 


\begin{tabular}{|c|c|c|c|c|c|c|c|c|}
\hline & 10 & 20 & 30 & 40 & 50 & 60 & 70 & 80 \\
\hline & .1 & & & & $\ldots 1$ & & .1 & \\
\hline AY621317.1 & CCCACAGAGC & ATCCATGAGG & TCATGGAGGT & ATTTCTGAAT & TTGAAGAAAA & TGTAATATAA & ATGCCGTTTT & АTCTGTACAG \\
\hline Sample_1 & $\cdots$ & & $\ldots$ & $\cdots$ & $\ldots \ldots \ldots$ & $\ldots \ldots \ldots$ & $\ldots$..... & . C.. \\
\hline Sample_2 & $\ldots \ldots \ldots$ & $\ldots$ & $\ldots \ldots \ldots$ & $\ldots \ldots \ldots$ & $\ldots \ldots \ldots$ & $\ldots \ldots \ldots$ & $\ldots$. R... & $\ldots$ \\
\hline Sample_3 & $\ldots \ldots$ & & $\ldots \ldots$ & & $\ldots \ldots \ldots$ & & $\ldots$..... &.$c \ldots \ldots$ \\
\hline Sample_4 & $\ldots \ldots \ldots$ & $\ldots \ldots \ldots$ & $\ldots \ldots \ldots$ & $\ldots$ & $\ldots \ldots \ldots$ & $\ldots \ldots \ldots$ & $\ldots \ldots \ldots$ & $\ldots$ \\
\hline Sample_5 & $\ldots \ldots \ldots$ & $\ldots \ldots \ldots$ & $\ldots \ldots \ldots$ & $\ldots \ldots \ldots \ldots$ & $\ldots \ldots \ldots$ & & $\ldots$. &.$c \ldots \ldots$ \\
\hline Sample_6 & . & $\ldots$ & $\ldots$ & . & $\ldots$ & $\ldots$ & $\ldots$..... & $\ldots$ \\
\hline \multirow[t]{3}{*}{ Sample_7 } & & & & & & ${ }_{\text {(a) }} w^{W} \cdots$ & $\begin{array}{c}\cdots A \\
\text { (b) }\end{array}$ & $\begin{array}{l}C \ldots \cdots \\
\text { (c) }\end{array}$ \\
\hline & 90 & 100 & $0 \quad 110$ & $0 \quad 12$ & $0 \quad 130$ & 140 & 150 & \\
\hline & $\ldots \ldots|\ldots|$ & $\begin{array}{l}\ldots|\ldots| \ldots \mid \\
\text { TGTAGATTCC }\end{array}$ & $\ldots \ldots|\ldots|$ & $\ldots \ldots|\ldots|$ & $\ldots,|\ldots \ldots|$ & $\ldots|\ldots|$ & $\ldots .|\ldots|$ & $\ldots \ldots|\ldots|$ \\
\hline Sample_1 & $\ldots \ldots \ldots \ldots$ & $\ldots$. . . . & $\begin{array}{l}\text { AGGGACTGCL } \\
\text {.A. . . . . }\end{array}$ & $\begin{array}{l}\text { IGLCACATAC } \\
\ldots \ldots \ldots \ldots\end{array}$ & $\ldots \ldots \ldots \ldots$ & ССттाтест & $\begin{array}{l}\text { GIAGLAGLTC } \\
\ldots \ldots \ldots \ldots\end{array}$ & $\begin{array}{c}\text { AGLAGATCL } \\
\ldots \ldots \ldots \ldots\end{array}$ \\
\hline Sample_2 & $\ldots$ & $\ldots R \ldots$ & R...... & & & & & \\
\hline Sample_-3 & $\ldots \ldots \ldots$ & ..G..... &.$A \ldots \ldots$ & $\ldots$ & $\ldots \ldots \ldots$ & $\ldots \ldots \ldots$ & . & $\ldots \ldots \ldots$ \\
\hline Sample_4 & $\ldots \ldots \ldots$ & $\ldots \ldots \ldots$ & $\ldots \ldots \ldots$ & $\ldots \ldots \ldots$ & $\ldots \ldots \ldots$ & $\ldots \ldots \ldots$ & $\ldots \ldots$ & $\ldots$ \\
\hline Sample_5 & $\ldots \ldots \ldots$ & $\ldots G \ldots \ldots$ &.$A \ldots \ldots$ & $\ldots \ldots \ldots$ & $\ldots \ldots \ldots$ & $\ldots \ldots \ldots$ & $\cdots$ & $\cdots$ \\
\hline Samp & $\ldots \ldots \ldots$ & ..G...... & .A. $\ldots \ldots$ & $\ldots \ldots \ldots$ & $\ldots \ldots \ldots$ & $\ldots$ & $\ldots$ & $\ldots$ \\
\hline \multirow[t]{3}{*}{ Sample_7 } & $\cdots$ & ${ }_{\text {(d) }}{ }^{\prime} \ldots$ & $\underset{(e)}{R} \ldots \ldots$ & & . & & & . \\
\hline & 170 & 180 & 190 & 20 & 210 & 220 & 230 & 240 \\
\hline & $\ldots|\ldots|$ & & $\ldots|\ldots|$ & $\ldots \ldots|\ldots|$ & $\ldots|\ldots|$ & $\ldots|\ldots|$ & $\cdot|\ldots|$. & \\
\hline AY621317.1 & CAGCCATGAG & GATTCTTTAC & СTGCTTTTCT & СТСтССтСтT & CCTGGCACTC & CAGGCTTCTC & CAGGTAAGAT & GAAAGAGGAA \\
\hline Samp & $\ldots \ldots \ldots$ & $\ldots \ldots \ldots$ & $\ldots \ldots \ldots$ & $\ldots \ldots \ldots$ & $\ldots \ldots \ldots$ & $\ldots$. & $\ldots \ldots \ldots$ & $\ldots$ \\
\hline Sample_2 & $\ldots \ldots \ldots$ & $\ldots \ldots \ldots$ & $\ldots \ldots \ldots$ & $\ldots \ldots \ldots$ & $\ldots \ldots \ldots$ & $\ldots Y \ldots$ & . & $\cdots$ \\
\hline Sample_3 & $\ldots$ & $\ldots \ldots$ & $\ldots \ldots \ldots$ & $\ldots$ & $\ldots \ldots \ldots$ & $\ldots$. $\ldots$ & $\cdots$ & $\ldots$ \\
\hline Sample_4 & $\ldots \ldots \ldots$ & $\ldots \ldots \ldots$ & $\ldots \ldots \ldots$ & $\ldots \ldots \ldots$ & $\ldots \ldots \ldots$ & $\ldots \ldots \ldots$ & $\ldots \ldots$ & $\ldots \ldots \ldots$ \\
\hline Samp & $\ldots \ldots \ldots$ & $\ldots \ldots \ldots$ & $\ldots \ldots \ldots$ & $\ldots \ldots$ & $\ldots \ldots \ldots$ & $\ldots$. & $\ldots \ldots$ & $\ldots \ldots \ldots$ \\
\hline Sample_6 & $\ldots \ldots \ldots$ & $\ldots \ldots \ldots$ & $\ldots \ldots \ldots$ & $\cdots \cdots$ & $\ldots \ldots \ldots$ & ..т.... & . & $\ldots$ \\
\hline \multirow[t]{3}{*}{ Sample_7 } & $\cdots$ & $\ldots$ & $\ldots$ & $\ldots$ & $\cdots$ & $\underset{\text { (f) }}{\cdots T}$ & & . \\
\hline & 250 & 260 & 270 & 28 & 290 & 300 & 310 & 320 \\
\hline & $\ldots|\ldots|$ & $\ldots .1$ & $\ldots|\ldots|$ & .. & $\ldots 1$ & $\ldots 1$ & $\ldots|\ldots|$ &. .1 \\
\hline AY 621 & TTAAAGGGGA & GGATAACGAC & TGGGTTATGG & GGAAGGGTTT & GCAGACCCGC & TTTGTGAGCT & САССТTТСАА & CGTGGCCAAA \\
\hline Sample_1 & $\ldots \ldots \ldots$ & $\ldots \ldots \ldots$ & $\ldots \ldots \ldots$ & $\ldots \ldots \ldots$ & ..... & $\ldots \ldots \ldots$ & $\ldots \ldots \ldots$ & $\ldots \ldots \ldots$ \\
\hline Sampl & $\ldots \ldots \ldots$ & $\ldots \ldots \ldots$ & $\ldots \ldots \ldots$ & $\ldots \ldots$ & $\ldots \ldots \ldots$ & $\ldots \ldots \ldots$ & $\ldots \ldots$ & $\ldots \ldots \ldots$ \\
\hline Sample_3 & $\ldots \ldots \ldots$ & $\cdots$ & $\cdots$ & $\cdots$ & $\ldots \ldots$. & . & . & $\ldots \ldots \ldots$ \\
\hline Samp & $\cdots \cdots \cdots$ & $\cdots \cdots$ & $\cdots$ & $\cdots$ & $\cdots$ & $\cdots$ & - & $\cdots$ \\
\hline Sample_5 & $\cdots \cdots \cdots$ & $\cdots \cdots \cdots$ & $\cdots \cdots \cdots$ & $\cdots \cdots \cdots$ & $\cdots \cdots$ & $\cdots \cdots \cdots$ & $\cdots \cdots \cdots$ & $\cdots \cdots$ \\
\hline Sample_6 & $\cdots$ & $\cdots$ & $\cdots$ & $\cdots$ & .......... & $\cdots \cdots$ & $\cdots$ & $\cdots$ \\
\hline \multirow[t]{3}{*}{ Sample_7 } & $\cdots$ & & $\ldots \ldots \ldots$ & $\cdots$ & $\begin{array}{r}\cdots \cdots_{(g)} \\
\quad\end{array}$ & $\ldots \ldots \ldots$ & $\cdots \cdots$ & $\ldots \ldots \ldots$ \\
\hline & 330 & 340 & 350 & 360 & 370 & 380 & 390 & \\
\hline & $\ldots|\ldots|$ & & $\ldots 1$ & & $\ldots \ldots|\ldots|$ & $\ldots 1$ & $|\ldots| \mid$ & $\ldots 1$ \\
\hline AY621317.1 & СССТCACAGC & AGTCCTTAAG & GCAGCTGAGT & GAGTGGAGCT & GCCTTGCCTT & GCAGAATCAG & AGGGAACTTG & GTTGCTGTTG \\
\hline Sampl & $\ldots \ldots \ldots$ & $\ldots \ldots \ldots$ & $\ldots \ldots \ldots$ & $\ldots \ldots \ldots$ & $\ldots \ldots \ldots$ & $\ldots \ldots \ldots$ & $\ldots \ldots \ldots k$ & $\ldots \ldots \ldots$ \\
\hline Sample_2 & $\ldots \ldots \ldots$. & $\cdots$ & $\ldots \ldots \ldots$ & $\cdots$ & $\cdots \cdots$ & $\cdots$ & $\cdots$ & \\
\hline Sample_3 & $\ldots k \ldots \ldots$ & $\ldots \ldots \ldots$ & $\ldots \ldots \ldots$ & $\ldots$ & $\ldots \ldots \ldots$ & $\ldots \ldots \ldots$ & $\ldots \ldots \ldots T$ & $\cdots$ \\
\hline Sample_4 & $\ldots \ldots \ldots$ & $\ldots \ldots \ldots$ & $\ldots \ldots \ldots$ & ... & $\ldots \ldots \ldots$ & & . & \\
\hline Sample_5 & $\ldots G \ldots \ldots$ & & & & $\cdots$ & & $\ldots \mathrm{T}$ & \\
\hline Sample_6 & $\ldots \ldots \ldots$ & $\ldots \ldots \ldots$ & $\ldots \ldots \ldots$ & $\cdots$ & $\ldots \ldots \ldots \ldots$ & $\ldots \ldots \ldots \ldots$ & $\cdots$ & \\
\hline \multirow[t]{2}{*}{ Sample_7 } & ${ }_{(h)}^{\ldots K} \ldots \ldots$ & & $\cdots$ & $\cdots$ & . & . & $\begin{aligned} \cdots \cdots \cdots & K \\
& (j)\end{aligned}$ & \\
\hline & $\ldots|\ldots|$ & & & & & & & \\
\hline AY621317.1 & TTGCAGGGTT & G & & & & & & \\
\hline Sample_1 & $\ldots \ldots \ldots$ & . & & & & & & \\
\hline Sample_2 & $\ldots \ldots \ldots$ & & & & & & & \\
\hline Sample_3 & $\ldots \ldots \ldots$ & & & & & & & \\
\hline Sample_4 & $\ldots \ldots \ldots$ & . & & & & & & \\
\hline Sample_5 & $\ldots \ldots \ldots$ & & & & & & & \\
\hline Sample_6 & $\ldots \ldots \ldots$ & - & & & & & & \\
\hline Sample_7 & $\ldots \ldots \ldots$ & & & & & & & \\
\hline
\end{tabular}

Figure 2. Partial sequence of AvBD2 gene in IPB-D1 chicken including 10 SNPs position, i.e. g.4843T>A (a), g.4853G $>$ A (b), g.4859T>C (c), g.4881A $>\mathrm{G}(\mathrm{d}), \mathrm{g} .4889 \mathrm{G}>\mathrm{A}(\mathrm{e}), \mathrm{g} .5002 \mathrm{C}>\mathrm{T}$ (f), g.5075C >T (g), g.5111T>G (h), g.5116G $>\mathrm{T}$ (i), and g.5177G $>\mathrm{T}(\mathrm{j})$ 
Table 2. Genotype frequency, allele frequency, and heterozygosityof AvBD2 gene in IPB-D1 chicken

\begin{tabular}{|c|c|c|c|c|c|c|c|c|}
\hline \multirow{3}{*}{$\frac{\text { SNP }}{\text { g. } 4843 \mathrm{~T}>\mathrm{A}}$} & \multirow{3}{*}{$\begin{array}{l}\mathrm{N} \\
47\end{array}$} & \multicolumn{3}{|c|}{ Genotype Frequency } & \multicolumn{2}{|c|}{ Allele Frequency } & \multirow{3}{*}{$\begin{array}{c}\text { Ho } \\
0.064\end{array}$} & \multirow{3}{*}{$\begin{array}{r}\mathrm{He} \\
0.062\end{array}$} \\
\hline & & TT & AT & AA & $\mathrm{T}$ & A & & \\
\hline & & 0.936 & 0.064 & 0 & 0.968 & 0.032 & & \\
\hline \multirow{2}{*}{ g. $4853 \mathrm{G}>A$} & \multirow{2}{*}{47} & GG & $\mathrm{AG}$ & AA & G & A & \multirow{2}{*}{0.191} & \multirow{2}{*}{$0.20^{7}$} \\
\hline & & 0.021 & 0.191 & 0.787 & 0.117 & 0.883 & & \\
\hline \multirow{2}{*}{ g. $4859 \mathrm{~T}>\mathrm{C}$} & \multirow{2}{*}{47} & TT & $\mathrm{TC}$ & $\mathrm{CC}$ & $\mathrm{T}$ & $\mathrm{C}$ & \multirow{2}{*}{0.170} & \multirow{2}{*}{0.22} \\
\hline & & 0.043 & 0.170 & 0.787 & 0.128 & 0.872 & & \\
\hline \multirow{2}{*}{ g. $4881 \mathrm{~A}>\mathrm{G}$} & \multirow{2}{*}{47} & AA & $\mathrm{AG}$ & GG & A & G & \multirow{2}{*}{0.234} & \multirow{2}{*}{0.268} \\
\hline & & 0.043 & 0.234 & 0.723 & 0.160 & 0.840 & & \\
\hline \multirow{2}{*}{ g. $4889 \mathrm{G}>A$} & \multirow{2}{*}{47} & GG & GA & AA & G & A & \multirow{2}{*}{0.255} & \multirow{2}{*}{0.25} \\
\hline & & 0.021 & 0.255 & 0.723 & 0.149 & 0.851 & & \\
\hline \multirow{2}{*}{ g. $5002 \mathrm{C}>\mathrm{T}$} & \multirow{2}{*}{47} & $\mathrm{CC}$ & $\mathrm{CT}$ & TT & $\mathrm{C}$ & $\mathrm{T}$ & \multirow{2}{*}{0.191} & \multirow{2}{*}{0.207} \\
\hline & & 0.021 & 0.191 & 0.787 & 0.117 & 0.883 & & \\
\hline \multirow{2}{*}{ g. $5075 \mathrm{C}>\mathrm{T}$} & \multirow{2}{*}{47} & $\mathrm{CC}$ & $\mathrm{CT}$ & TT & $\mathrm{C}$ & $\mathrm{T}$ & \multirow{2}{*}{0.362} & \multirow{2}{*}{0.50} \\
\hline & & 0.319 & 0.362 & 0.319 & 0.500 & 0.500 & & \\
\hline \multirow{2}{*}{ g. $5111 \mathrm{~T}>\mathrm{G}$} & \multirow{2}{*}{47} & TT & TG & GG & $\mathrm{T}$ & G & \multirow{2}{*}{0.277} & \multirow{2}{*}{0.323} \\
\hline & & 0.660 & 0.277 & 0.064 & 0.798 & 0.202 & & \\
\hline & & GG & GT & TT & G & $\mathrm{T}$ & & \\
\hline 8.011008 & 41 & 0.723 & 0.255 & 0.021 & 0.851 & 0.149 & 0.253 & 0.234 \\
\hline a $5177 \mathrm{G}>\mathrm{T}$ & 47 & GG & GT & TT & G & $\mathrm{T}$ & 0362 & 0400 \\
\hline 8.01780 & 41 & 0.298 & 0.362 & 0.340 & 0.479 & 0.521 & 0.002 & 0.473 \\
\hline
\end{tabular}

$\mathrm{N}$ = total sample; Ho=observed heterozygosity; He=expected heterozygosity

g.4881A>G, g.4889G $>$ A, g.5002C $>\mathrm{T}$, and g.5075C $>\mathrm{T}$. While the g.4843T $>$ A, g.5111T $>\mathrm{G}$, g.5116G $>\mathrm{T}$, and g.5177G $>\mathrm{T}$ mutations indicated a transversion mutation. Transition mutations are changes in nucleotide substitution that have the same structure, such as substitution between purine with purine $(A>G)$ or pyrimidine with pyrimidine $(\mathrm{C}>\mathrm{T})$. This transition mutation does not change hydrogen bonds, while a transversion mutation is a change in nucleotide substitution that has a different structure, ie. in purine to pyrimidine, or pyrimidine to purine (Luo et al. 2016). Transitions are more conservative in their effects on proteins than transversions that may cause a weaker effect of transitions on structure and function of gene regulation (Stoltzfus \& Norris 2016). Figure 2 describes the AvBD2 gene sequence alignment in IPB-D1 chickens.

According to Table 1, there are 3 mutations located in exon 2, i.e g.4881 A > G, g.4889G $>A$, and g.5002C $>$ T. Both g.4881A $>\mathrm{G}$ and g.4889G $>\mathrm{A}$ located in the noncoding region, which are not translated into amino acids. Meanwhile, the g.5002C $>\mathrm{T}$ is located in the coding region. This $\mathrm{g} .5002 \mathrm{C}>\mathrm{T}$ mutation also alters the amino acid Alanine to Valine. This missense mutation was also reported by Morammazi \& Habibi (2017).

Nie et al. (2018) mentioned that Valine is one of 3 branched chain amino acids (BCAAs), including leucine (Leu) and isoleucine (Ile). These BCAAs play an important role in the regulation of energy homeostasis, nutrient metabolism, immunity and disease resistance in humans and animals (Nie et al. 2018). The non-synonymous mutation of the AvBD2 gene plays an important role in the antimicrobials found in different muscle tissues (Hellgren et al. 2010). This Alanine to Valine mutation needs to be further studied to understand its effect on disease resistance in chickens.

Avian beta defensin gene family has been reported to have strong association with chicken bacterial resistance. A total of 5 SNPs at the AvBD5 gene were reported to be associated with Salmonella susceptibility in China local chicken (Zhang et al. 2020). Using same chicken population, four SNPs at the AvBD14 gene were also associated with Salmonella susceptibility (Zhang et al. 2020). To our knowledge, no studies has been reported the association between the AvBD2 gene with bacterial resistance in chicken. 


\section{Polymorphism Information of the AVBD2 Gene}

Table 2 shows a total of 10 SNPs found in this study, and each SNP has 2 alleles and 3 genotypes except g.4843T $>$ A which has 2 genotypes (TT and AT) of 2 alleles (A and T). Individuals with genotype AA were not found at g.4843T >A. All alleles on each SNP had a frequency higher than $0.01(1 \%)$, which indicated that all SNPs in the AVBD2 gene were polymorphic in the IPB-D1 chicken population. In accordance with the explanation of Nei \& Kumar (2000) that a population with 2 or more alleles with a frequency value of more than 0.01 or $1 \%$ indicates that the gene is polymorphic. The highest allele frequency at g.4843T $>\mathrm{A}$, g.4853G $>A, \quad$ g.4859T $>C, \quad$ g.4881A $>$ G, g.102G $>A$, g. $5002 \mathrm{C}>\mathrm{T}$, g. $5111 \mathrm{~T}>\mathrm{G}$, g. $5116 \mathrm{G}>\mathrm{T}$, and g. $5177 \mathrm{G}>\mathrm{T}$ are the T, A, C, G, A, T, T, G and T alleles, respectively. Whereas at the g.5075C $>\mathrm{T}$, both $\mathrm{C}$ and $\mathrm{T}$ alleles have the equal frequency (0.5).

Heterozygosity value calculations were carried out to determine how high the level of genetic diversity at a locus based on allele frequencies (Wang et al. 2015). All loci were found to have Ho values lower than 0.5 , this indicated that all loci had a low level of diversity (Allendorf et al. 2013). Apart from being an indicator of the level of diversity, the comparison of $\mathrm{Ho}$ and $\mathrm{He}$ is used to indicate the occurrence of that mentioned by Allendorf et al. (2013). Of the ten loci found, 7 loci had lower Ho values than He, i.e g.4853G $>$ A, g.4859T $>$ C, g. $4881 \mathrm{~A}>\mathrm{G}$, g. $5002 \mathrm{C}>\mathrm{T}$, g. $5075 \mathrm{C}>\mathrm{T}$, g. $5111 \mathrm{~T}>\mathrm{G}$, and g. $5177 \mathrm{G}>\mathrm{T}$. Ho value which is lower than He may indicate the occurrence of inbreeding in the IPB-D1 chicken population.

Previously, it was explained that the mutation of the nucleotide $\mathrm{C}$ to $\mathrm{T}$ in the missense mutation g.5002C $>\mathrm{T}$ in exon 2 is known to change the amino acid Alanine to Valine. Results of the amino acid sequence prediction showed that the $\mathrm{T}$ allele will produce the amino acid Valine which plays an important role in immunity and disease resistance. Based on Table 2, the $\mathrm{T}$ allele at the g.5002C $>\mathrm{T}$ locus has a much higher frequency $(0.883)$ than the $C$ locus $(0.117)$. This indicates that most of the IPB-D1 chicken population tends to have good resistance. However, this proposal needs to be strengthened by further studies.

\section{CONCLUSION}

In this study, a total of 10 SNPs were found in the intron 1 to intron 2 of the AvBD2 gene in the IPB-D1 chicken population. All SNPs are polymorphic. A total of 7 SNPs were found in intron 1 (g.4843T $>A$, g.4853G $>$ A, g.4859T $>C)$ and intron $2($ g.5075C $>\mathrm{T}$, g. $5111 \mathrm{~T}>\mathrm{G}$, g. $5116 \mathrm{G}>\mathrm{T}$ and g. $5177 \mathrm{G}>\mathrm{T})$. While the other 3 SNPs were found in exon $2(\mathrm{~g} .4881 \mathrm{~A}>\mathrm{G}$, g. $4889 \mathrm{G}>\mathrm{A}$, g.5002C $>\mathrm{T})$. A missense mutation was found in $\mathrm{g} .5002 \mathrm{C}>\mathrm{T}$ which changed the amino acid Ala to $\mathrm{Val}$. This g.5002C $>\mathrm{T}$ has the potential as a candidate marker for disease resistance in IPB-D1 chickens.

\section{ACKNOWLEDGEMENT}

Authors thank to Ministry of Research and Education, RI for the financial support for this study (World Class Research with contract no: 3815/IT3.L1/PN/2020).

\section{REFERENCES}

[DGAHP] Directorate General of Animal Health and Production. 2020. Livestock and Animal Health Statistics 2020. Jakarta (Indones): Directorate General of Animal Health and Production.

Al-Habib MF, Murtini S, Gunawan A, Ulupi N, Sumantri C. 2020. Polymorphism of CD1B gene and its association with yolk immunoglobulin (IgY) concentration and Newcastle Disease antibody titer in IPB-D1 chicken. Trop Anim Sci J. 43:197-204. DOI: 10.5398/tasj.2020.43.3.197.

Allendorf FW, Luikart GH, Aitken SN. 2013. Conservation and the Genetics of Population. 2nd ed. West Sussex (UK): Wiley-Blackwell.

Bagnicka E, Strzałkowska N, Jóźwik A, Krzyżewski J, Horbańczuk J, Zwierzchowski L. 2010. Expression and polymorphism of defensins in farm animals. Acta Biochim Pol. 57:487-497. DOI: 10.18388/abp.2010_2434.

Derache C, Meudal H, Aucagne V, Mark KJ, Cadène M, Delmas AF, Lalmanach A-C, Landon C. 2012. Initial Insights into Structure-Activity Relationships of Avian Defensins. J Biol Chem. 287:7746-7755. DOI: 10.1074/jbc.M111.312108.

Hall T, Biosciences I, Carlsbad C. 2011. BioEdit: An important software for molecular biology. GERF Bull Biosci. 2:60-61.

Hazlett L, Wu M. 2011. Defensins in innate immunity. Cell Tissue Res. 343:175-188. DOI: 10.1007/s00441-0101022-4.

Hellgren O, Sheldon BC, Buckling A. 2010. In vitro tests of natural allelic variation of innate immune genes (avian $\beta$-defensins) reveal functional differences in microbial inhibition. J Evol Biol. 23:2726-2730. DOI: 10.1111/j.1420-9101.2010.02115.x.

Hong YH, Song W, Lee SH, Lillehoj HS. 2012. Differential gene expression profiles of $\beta$-defensins in the crop, intestine, and spleen using a necrotic enteritis model in 2 commercial broiler chicken lines. Poult Sci. 91:10811088. DOI: $10.3382 / p s .2011-01948$.

Kim WH, Lillehoj HS. 2019. Immunity, immunomodulation, and antibiotic alternatives to maximize the genetic potential of poultry for growth and disease response. 
Anim Feed Sci Technol. 250:41-50. DOI: 10.1016/j.anifeedsci.2018.09.016.

Kumar S, Stecher G, Li M, Knyaz C, Tamura K. 2018. MEGA X: Molecular evolutionary genetics analysis across computing platforms.Battistuzzi FU, editor. Mol Biol Evol. 35:1547-1549. DOI: 10.1093/molbev/msy096.

Liu C, Jiang L, Liu L, Sun L, Zhao W, Chen Y, Qi T, Han Z, Shao Y, Liu S, Ma D. 2018. Induction of Avian $\beta$ Defensin 2 is possibly mediated by the p38 MAPK signal pathway in chicken embryo fibroblasts after Newcastle Disease virus infection. Front Microbiol. 9:751. DOI: 10.3389/fmicb.2018.00751

Lu S, Peng K, Gao Q, Xiang M, Liu H, Song H, Yang K, Huang H, Xiao K. 2014. Molecular cloning, characterization and tissue distribution of two ostrich $\beta$ defensins: AvBD2 and AvBD7. Gene. 552:1-7. DOI: 10.1016/j.gene.2014.08.019.

Luo G-H, Li X-H, Han Z-J, Zhang Z-C, Yang Q, Guo H-F, Fang J-C. 2016. Transition and Transversion Mutations Are Biased towards GC in Transposons of Chilo suppressalis (Lepidoptera: pyralidae). Genes (Basel). 7:72. DOI: 10.3390/genes7100072.

Morammazi S, Habibi H. 2017. Sequence variation in GAL1 and GAL2 genes in Khuzestan local chickens. Eur Online J Nat Soc Sci. 4:508-515.

Nei Masatoshi, Kumar S. 2000. Molecular Evolution and Phylogenetics. 1st ed. New Jersey (USA): Oxford University Press.

Nei M, Kumar S. 2000. Molecular Evolution and Phylogenetics. New York (US): Oxford Univ Pr.

Nie C, He T, Zhang W, Zhang G, Ma X. 2018. Branched Chain Amino Acids: Beyond Nutrition Metabolism. Int J Mol Sci. 19:954. DOI: 10.3390/ijms19040954.

Shimizu M, Nii T, Isobe, N, Yoshimura Y. 2020. Effects of avian infectious bronchitis with Newcastle disease and Marek's disease vaccinations on the expression of tolllike receptors and avian $\beta$-defensins in the kidneys of broiler chicks. Poult Sci. 99:7092-7100. DOI: 10.1016/j.psj.2020.08.071.
Stoltzfus A, Norris RW. 2016. On the causes of evolutionary transition: transversion bias. Mol Biol Evol. 33:595602. DOI: $10.1093 / \mathrm{molbev} / \mathrm{msv} 274$.

Sumantri C, Khaerunnisa I, Gunawan A. 2020. The genetic quality improvement of native and local chickens to increase production and meat quality in order to build the Indonesian chicken industry. IOP Conf Ser Earth Environ Sci. 492:012099. DOI: 10.1088/17551315/492/1/012099.

Susanti F, Murtini S, Wibawan I. 2020. Immune response of IPB-D1 chickens with TLR4 genes against Salmonella enteritidis bacterial infection. J Vet. 21:208-215.

Terada T, Nii T, Isobe N, Yoshimura Y. 2018. Changes in the expression of Avian $\beta$-defensins (AvBDs) and proinflammatory cytokines and localization of AvBD2 in the intestine of broiler embryos and chicks during growth. J Poult Sci. 55:280-287. DOI: 10.2141/jpsa.0180022

Wang J, Raskin L, Samuels DC, Shyr Y, Guo Y. 2015. Genome measures used for quality control are dependent on gene function and ancestry. Bioinformatics. 31:318-323. DOI: 10.1093/bioinformatics/btu668

Wilson SS, Wiens ME, Smith JG. 2013. Antiviral Mechanisms of Human Defensins. J Mol Biol. 425:4965-4980. DOI: 10.1016/j.jmb.2013.09.038.

Xiao Y, Hughes AL, Ando J, Matsuda Y, Cheng J-F, SkinnerNoble D, Zhang G. 2004. A genome-wide screen identifies a single $\beta$-defensin gene cluster in the chicken: implications for the origin and evolution of mammalian defensins. BMC Genomics. 5:56. DOi: 10.1186/1471-2164-5-56.

Zhang LY, Huang MY, Li Y, Chen DZ, Shi X. 2020. Association of three beta-defensin gene (AvBD4, AvBD5, AvBD14) polymorphisms with carrier-state susceptibility to salmonella in chickens. Br Poult Sci. 61:357-365. DOI: 10.1080/00071668.2020.1752913.

Zhao L, Yang M, Zhang M, Zhang S. 2014. Expression, Purification, and In Vitro Comparative Characterization of Avian Beta-Defensin-2, -6, and -12. Avian Dis. 58:541-549. DOI: 10.1637/10848-042014-Reg.1. 